\title{
Pour une muséologie contractuelle
}

In favour of a contractual museology

\section{Bernard Deloche}

\section{CpenEdition}

\section{Journals}

Édition électronique

URL : http://journals.openedition.org/iss/587

DOI : $10.4000 /$ iss. 587

ISSN : 2306-4161

\section{Éditeur}

ICOM - International Council of Museums

\section{Édition imprimée}

Date de publication : 1 juin 2015

Pagination : 83-93

ISSN : 2309-1290

\section{Référence électronique}

Bernard Deloche, « Pour une muséologie contractuelle », ICOFOM Study Series [En ligne], 43a | 2015

mis en ligne le 06 février 2018, consulté le 19 avril 2019. URL : http://journals.openedition.org/iss/587 ; DOI : 10.4000/iss.587 


\title{
Pour une muséologie contractuelle
}

\author{
Bernard Deloche \\ Professeur émérite à l'Université Jean Moulin (Lyon 3) - \\ France
}

S'il n'y a pas de définition précise du " champ du savoir » auquel s'appliquerait la muséologie, c'est simplement parce que la muséologie n'est pas une science et qu'elle ne produit pas de connaissance ni, a fortiori, de savoir (connaissance absolue et définitive). Ce constat résulte in fine de débats internationaux qui ont animé les muséologues dans les années 1970 à 1990, notamment au sein de l'ICOFOM (les articles rédigés dans MuWoP en 1980). Les arguments étant irréfutables, la discussion semble désormais close (Hudson, 1997). Mais la double question du domaine d'application de la muséologie et de sa mission demeure entière.

Aussi convient-il d'abord de délimiter exactement le champ muséal ou la spécificité propre au musée, afin de comprendre de quelle nature est l'activité de cette institution et, par là, quel est le statut de la discipline qui en a fait son objet. Ce champ, qui constitue un point de vue spécifique de relation de l'homme avec la réalité (Gregorova, 1980 ; Stransky, 1980), consiste en une opération de documentation (collecter, analyser, montrer) sensible (à la différence des bibliothèques) et peut porter sur n'importe quelle catégorie d'objets (Desvallées, 1980).

Puisque la muséologie n'est ni une science ni un mode de connaissance, on peut considérer qu'il s'agit d'une éthique, une instance de choix des valeurs auxquelles on soumet l'activité du musée. De fait, depuis la naissance de l'institution, ces valeurs ont considérablement évolué pour nous conduire, par exemple, du musée des reliques aux actuels musées de société. Ces choix successifs supposent bien sûr une connaissance précise de l'histoire et du mode de fonctionnement des musées. Mais la muséologie ne saurait se réduire à cette connaissance, car il faut savoir où l'on va, ce qui relève d'enjeux que l'on pourra qualifier de philosophiques (Desvallées, 1980 ; Spielbauer, 1987). De tels choix ne peuvent s'imposer du dehors et ne sauraient résulter que d'une série de consensus, eux-mêmes historiques et contractuels.

Le texte de présentation du symposium indique à l'axe 9 que «la muséologie s'est progressivement développée comme un champ de savoir [et que...], jusqu'à présent, il n'y a pas de définition précise du champ de la muséologie ". C'est ce point que je voudrais reprendre pour le discuter. La question est complexe dans la mesure où elle mêle divers problèmes appartenant à des niveaux de compréhension différents : l'objet de la muséologie comme discipline (s'agit-il, par exemple, de la connaissance de l'histoire et du fonctionnement des musées ?), la mission du musée lui-même (est-il un lieu de culte ou bien un outil de transmission voire de communication ?), la question du champ et celle du savoir (est-on dans l'ordre de la connaissance ou au contraire dans celui de l'action ?) et enfin celle de la légitimité même de l'expression "champ de savoir » en matière de muséologie. 
Nous allons voir que, s'il y a bien un champ muséal clairement défini, celui-ci résulte d'une série de choix historiques et n'est pas de l'ordre du savoir mais s'inscrit plutôt dans le cadre d'une éthique.

\section{Le champ muséal, un point de vue spécifique sur la réalité}

II convient d'abord de délimiter exactement le champ muséal - ou, plus simplement, "le muséal » - afin de comprendre quelle est la nature de l'activité de cette institution qu'on appelle musée.

Ce champ constituerait une forme spécifique de notre relation avec la réalité. C'est ainsi que, dès les années 1980, les muséologues tchécoslovaques ont en partie bouleversé les fondements théoriques de la muséologie en refusant de considérer le musée comme l'objet de la muséologie et en introduisant une dimension plus globale, à caractère ontologique au moins nominalement, celle de la " relation spécifique de l'homme avec la réalité " (Gregorova, 1980). II s'agit d'une sorte de révolution copernicienne par laquelle l'objet de la muséologie se ramènerait à cette relation spécifique, dont le musée ne serait qu'une matérialisation particulière. Si le thème n'était pas vraiment nouveau, en revanche son application au musée l'était totalement. Jusqu'alors on parlait par exemple du politique, $d u$ social, $d u$ culturel, $d u$ scolaire, etc., toujours précédés de l'article masculin pour distinguer le champ de l'activité qui lui correspond (le champ politique distingué de l'activité politique, le champ social distingué de la vie sociale, etc.). Ces différents champs ou plans étaient considérés comme autant de modes de relation avec la réalité à la fois originaux et irréductibles les uns aux autres. Et il s'agit bien d'un champ au sens où, au même titre que les autres champs, le muséal est par définition sans limites assignables. Comme on a pu dire que tout est politique, il est également permis de dire que tout est muséal, car " le champ d'étude devient infini [...]. Tout est musée. », (Desvallées, 1987, p. 6). Bref le musée n'a pour limites que celles qu'il se donne (Desvallées, 1994, p. 84). Le propre d'un champ est qu'il renvoie à un point de vue et qu'il est, comme un faisceau lumineux, défini plus par son angle de projection que par la nature ou le nombre des objets sur lesquels il se projette.

Toutefois, traiter le muséal comme caractérisant une relation spécifique de l'homme avec la réalité était une démarche assez risquée, car on peut légitimement se demander si le domaine muséal est bien inscrit une fois pour toutes dans la réalité puisque sa dimension historique saute aux yeux du fait que le musée n'a pas toujours existé et qu'il est né officiellement, après diverses ébauches notamment en Grande-Bretagne dès le $\mathrm{XVII}{ }^{e}$ siècle (Ashmolean Museum d'Oxford), sous la Révolution française avec la fondation par la Convention des quatre premiers musées entre 1793 et 1795 .

Mais les muséologues tchécoslovaques ont, de façon pour le moins paradoxale ${ }^{58}$, su intégrer ce caractère historique du muséal tout en lui conservant d'une certaine manière un statut ontologique. Car il ne s'agit que d'un point de vue conçu par l'homme et donc, comme tout point de vue, il est relatif par définition ${ }^{59}$. Le muséal, de même que

\footnotetext{
${ }^{58}$ Position paradoxale parce que la dimension ontologique désigne une instance inscrite dans le réel, tandis que l'approche historique est anthropologique et ne renvoie qu'à l'homme et à sa manière d'appréhender le réel.

${ }^{59}$ Point de vue qui n'est pas inscrit dans le réel comme l'étaient, par exemple pour Pascal, les « Trois ordres », l'ordre des corps, l'ordre des esprits et l'ordre des cœurs,
} 
les autres champs évoqués plus haut à titre d'exemples, s'inscrit dans une démarche exclusivement anthropologique dont le caractère est plus pragmatique que théorique. C'est en effet l'humanité qui, au cours de son histoire, s'est penchée de cette manière sur le monde qui l'entoure ; ce sont les hommes, et eux seuls, qui ont défini des types de relations avec le réel.

Quant à sa qualification et à son contenu, ce point de vue, spécifique et propre au musée, consiste en une opération documentaire, qui se traduit par trois fonctions essentielles (collecter, analyser, montrer) : "Le musée est une institution qui applique et réalise le rapport spécifique homme-réalité, consistant dans la collection et la conservation conscients et systématiques et l'utilisation scientifique, culturelle et éducative des objets inanimés, matériels, mobiles (notamment tridimensionnels) qui documentent le développement de la nature et de la société. " (Gregorova, 1980, p. 20-21). Et, point capital, cette documentation est sensible, c'est-à-dire en principe accessible à nos cinq sens (voir, entendre, toucher, humer, goûter), en principe seulement, car très rares sont les musées qui vous proposent de découvrir des odeurs et des saveurs ; quant au toucher il est le plus souvent banni des comportements du visiteur et considéré comme sacrilège. Cette dimension sensible et concrète permet de distinguer le point de vue muséal de celui des bibliothèques, par exemple, qui proposent une documentation médiatisée par l'écrit, c'est-à-dire par un support à caractère conventionnel qui demande d'être appris. C'est ainsi qu'un tout jeune enfant, qui ne sait pas encore lire, pourra tirer profit d'une visite au musée, alors que dans une bibliothèque il ne trouvera rien qui soit à sa portée en dehors des livres d'images.

\section{Mais ce champ muséal n'est pas de l'ordre de la connaissance}

II y a donc bien un champ muséal historiquement conçu et construit par les hommes, mais ce champ est-il un " champ de savoir », c'està-dire d'ordre cognitif ou gnoséologique, comme l'affirmaient les muséologues tchécoslovaques ? En effet, si Z. Z. Stransky estimait que « la muséologie n'a pas sa place dans le système existant des sciences » (Stransky, 1980, p. 43), il ne s'agissait pour lui que d'une question de temps pour une discipline émergente et en cours de constitution, c'est-à-dire " une nouvelle discipline scientifique avec son objet d'étude »(Gregorova, 1980, p. 21).

Il me semble au contraire que, si le « champ du savoir » auquel s'appliquerait la muséologie n'a pas de définition précise, c'est tout simplement parce que la muséologie n'est pas une science et qu'elle ne produit pas de connaissance ni, a fortiori, de savoir (connaissance absolue et définitive). Ma position est donc, sur ce point, diamétralement opposée à celle des théoriciens tchécoslovaques, bien que je reconnaisse l'importance de la "relation spécifique » qui fait l'originalité propre du musée.

Rappelons brièvement pourquoi la muséologie n'est pas une science $^{60}$. Comme elle n'entre pas dans les sciences de la nature et

qualitativement cloisonnés c'est-à-dire présentant une différence de nature et non de simple degré (Pensées, $n^{\circ} 793$, éd. Brunschvicg).

${ }^{60}$ La teneur de cette argumentation a fait l'objet d'une leçon à l'École internationale d'été de muséologie (ISSOM) de Brno le $1^{\text {er }}$ septembre 1995, texte publié en 1999 dans ISS 31 sous le titre «Muséologie et philosophie » (Deloche, 1999) et 
ne pourrait être assimilée légitimement qu'aux sciences humaines ou sociales, pour côtoyer par exemple la sociologie, l'ethnologie, la psychologie, la linguistique ou l'économie, etc., la question est de savoir si elle s'inscrit normalement parmi ces disciplines. Mais une première constatation permet déjà de douter de la parfaite similitude de la muséologie avec les sciences de l'homme. Si le musée luimême est une forme institutionnelle possible d'une démarche scientifique active, c'est-à-dire un véritable outil de science, il s'apparente de plein droit à d'autres institutions qui lui sont comparables, comme l'école, l'hôpital ou la prison, institutions secondaires qui médiatisent des besoins sociaux donc artificiels par opposition aux institutions primaires qui médiatisent des besoins biologiques naturels. Ces institutions réunissent des êtres humains pour les instruire, les soigner et les surveiller. Le musée est une institution analogue, qui rassemble des objets ou leurs substituts pour les analyser et les présenter à un public. La muséologie s'est ainsi assez spontanément définie comme la discipline chargée d'étudier la formation, l'évolution et la diversification de cette institution qu'est le musée. Mais, alors qu'il n'existe pas de discipline autonome traitant de l'école, de l'hôpital ou de la prison, et seulement des disciplines relatives aux fonctions de ces institutions (la pédagogie, la clinique, la criminologie, par exemple), on s'attendrait normalement à voir se développer en parallèle - et à la place de la muséologie - une science générale de l'information et de la communication par des médias sensibles (dont fait partie le musée). Si cette dernière discipline existe effectivement, elle ne se confond nullement avec la muséologie car son champ d'application est beaucoup plus vaste. On a pu imaginer, par exemple, une société sans école (Ivan Illitch) ou une école autogérée (Alexander S. Neill), mais il n'est pas né de ces travaux une discipline autonome, une science, chargée d'étudier le scolaire. Or, chose étrange, la muséologie se présente empiriquement et de façon inattendue comme une métathéorie concentrée sur l'institution ou sur ses épigones et certains muséologues revendiquent pour elle le statut de science. Cette étrange dissymétrie par rapport aux institutions de même rang conduit à se demander pourquoi le musée serait la seule institution secondaire à s'être dotée d'une discipline propre pour laquelle l'institution primerait sur la fonction. Dans cette perspective, la muséologie serait une sorte de gardien moral de l'institution; c'est d'ailleurs ainsi qu'elle est très souvent comprise par les plus rétrogrades des conservateurs. II est bien évident que ce rôle n'est assurément pas assimilable à une tâche scientifique !

Mais surtout, la muséologie ne répond à aucun des critères épistémologiques d'une science de l'homme, à savoir la méthode de modélisation, le caractère cognitif - ou gnoséologique pour reprendre le terme de Stransky - et l'objectivité.

a) La muséologie ne pratique pas scientifiquement la méthode privilégiée des sciences de l'homme qu'est la modélisation. II n'y a pas en muséologie d'équivalent de la théorie des jeux en économie ou des structures de la parenté en anthropologie. Je pense d'ailleurs que si de tels modèles n'existent pas vraiment en muséologie c'est simplement parce que cette discipline est la théorisation d'une institution créée délibérément et consciemment par l'homme en vue

partiellement repris en 2001 dans Le musée virtuel (Deloche, 2001), mais tout cela ne semble pas avoir été réellement entendu puisque l'on trouve aujourd'hui encore des tenants de la muséologie comprise comme une science. Je dirais, pour paraphraser Horace, "Ter repetita placent». On ne s'étonnera donc pas de retrouver ici certains passages déjà publiés. 
de fins déterminées. La modélisation ne pourra être alors qu'illustrative ou normative mais non explicative.

b) La muséologie ne génère aucun contenu de connaissance. Toute science est un outil d'explication et de prévision des phénomènes fondé sur le postulat du déterminisme. Certes, cette rigueur est-elle moindre dans les sciences de l'homme qui ne sont pas des sciences exactes, mais qui tendent seulement à le devenir notamment par recours aux statistiques. Dans ces sciences, la connaissance véritable s'enracine dans la récurrence du modèle, c'est-à-dire dans la possibilité d'appliquer de façon féconde le même modèle à un nombre illimité de cas afin de prévoir les phénomènes. Or, rien de tel en muséologie, car le musée est déjà par lui-même un outil de connaissance (la documentation sensible). En revanche, comme méta-discipline, la muséologie ne livre aucun contenu de connaissance ; elle est seulement une réflexion sur une démarche de connaissance. Comme l'épistémologie, elle traite des fondements de la science, mais elle n'est pas une science au deuxième degré.

c) La muséologie n'est pas non plus une discipline objective ; elle ne respecte pas l'objet dans sa phénoménalité et ne cesse au contraire d'intervenir sur lui pour le modifier, car le musée est toujours à construire et à transformer. La muséologie n'étudie pas l'évolution des musées de l'extérieur tout en se gardant de modifier ce qu'elle analyse. Et si elle le modifie ce n'est pas pour mieux le connaître, ce qui serait une forme d'expérimentation, mais seulement pour mieux agir par son intermédiaire. Bref, le musée n'est pas pour la muséologie un phénomène à comprendre ou à expliquer, mais une institution ou un ensemble d'outils à définir et à corriger. À ce titre, dans la mesure où elle tend à édicter des règles, la muséologie s'apparenterait plutôt aux disciplines normatives.

Pour toutes ces raisons, il ne m'apparaît donc pas concevable d'assimiler la muséologie à une science, pas même à une science en cours d'élaboration, car elle ne répond vraiment à aucun des critères qui commandent la définition et l'exercice des sciences de l'homme. Ce constat résulte in fine de débats internationaux qui ont animé les muséologues dans les années 1970 à 1990 (notamment au sein de I'ICOFOM avec la publication de DoTraM-MuWoP $\mathrm{n}^{\circ} 1$ en 1980). Les arguments étant irréfutables, la discussion semble désormais close, et c'est ainsi que, pour Kenneth Hudson par exemple, «la muséologie n'est pas et ne peut pas être une science, pas plus que l'éducation ou la cuisine ne le peuvent, et toute tentative pour prouver le contraire est stupide, erronée et malhonnête " (Hudson, 1997, p. 108). Voilà qui est on ne peut plus clair et sans appel. Mais la double question de la mission de la muséologie et de son domaine d'application demeure entière.

\section{Moins bien cerné que celui du musée lui-même, le champ de la muséologie est d'ordre éthique}

Si la muséologie n'est ni une science ni un mode de connaissance, comment la caractériser? Dès les années 1990, j'ai proposé de considérer qu'il s'agissait d'une éthique, c'est-à-dire du choix des valeurs auxquelles on soumet l'activité du musée désormais reconnu comme n'étant qu'un simple moyen (Desvallées, 1989, p. 358). De fait, ces valeurs ont considérablement évolué au cours de l'histoire depuis la naissance de l'institution, pour nous conduire par exemple du musée reliquaire aux actuels musées de société. Ce choix de 
valeurs suppose bien sûr une connaissance précise du mode de fonctionnement des musées et de son histoire, mais il ne saurait s'y réduire, car il faut savoir où l'on va et cela relève d'enjeux que l'on pourra qualifier de philosophiques. Alors que l'institution du musée gère la documentation sensible, la muséologie est un organe de décision et c'est à elle de dire à quelles fins va servir cette documentation sensible. Voilà ce qui, selon moi, lui confère une importance considérable.

Ainsi comprise, la muséologie est étroitement liée aux fondements de l'action dont elle détermine la finalité ${ }^{61}$. Elle pose en particulier ces questions majeures : pourquoi montrer, quels objets, à qui et dans quelles conditions ${ }^{62}$ ? Elle intervient dans la définition des objectifs du musée, son rôle étant de fixer le sens de l'institution. Et, si elle n'est pas une science, elle doit être comprise comme le principe d'une " activité ", c'est-à-dire d'une « façon d'agir consciente [...]. Ce qui est essentiel pour une activité pratique, écrit Z. Z. Stransky, c'est qu'il s'agit d'une action consciente. L'agent de l'activité doit tout d'abord se rendre compte du but, de la raison de l'activité déployée ». Paradoxalement, c'est Z. Z. Stránský, l'un des grands défenseurs de l'avenir scientifique de la muséologie, qui nous engage sur cette voie pourtant étrangère à la démarche scientifique : « L'activité muséale et le musée comme institution, écrit-il, ne sont cependant pas eux-mêmes le but mais le moyen. Notre intention gnoséologique ne peut donc pas s'arrêter auprès des moyens mais doit viser à découvrir les buts qui doivent être atteints à l'aide de ces moyens. » (Stransky, 1986).

Nous touchons ici à l'essentiel. Si le musée peut être considéré avec juste raison non seulement comme un instrument didactique mais aussi comme un très puissant outil de connaissance, il n'est jamais qu'un moyen au service d'autre chose, et c'est à une métathéorie, en l'occurrence la muséologie, qu'il revient d'en déterminer les fins. Ainsi comprise, la muséologie n'est rien d'autre qu'une théorie élaborée en vue d'une pratique, donc une philosophie pratique, non pas seulement un "travail pratique du musée » au sens d'une activité quotidienne et banale d'organisation ou d'une technique de mise en scène et de présentation des expôts, à quoi se réduit peut-être la muséographie, mais bien la détermination par la raison d'objectifs axiologiques, c'est-à-dire de règles d'action.

Le musée est une institution diversifiée et réformable, dont on peut fixer les objectifs et sur laquelle il est possible d'agir pour la transformer. II n'y a donc pas un modèle normatif unique et idéal du musée qu'il faudrait à tout prix respecter ou retrouver, comme on l'a cru longtemps lorsqu'on le pensait soumis aux seules valeurs de l'humanisme occidental (ethnocentrisme encore récemment dénoncé à propos du musée du quai Branly). Mais on est plutôt à la recherche d'une institution - ou d'un faisceau d'institutions - capable de répondre à des besoins spécifiques de la société humaine. Voilà pourquoi il est possible de parler d'éthique. Contrairement à la

\footnotetext{
${ }^{61}$ Le titre du séminaire de l'École du Louvre en mars 1983, Quels musées, pour quelles fins, aujourd'hui ? était de ce point de vue tout à fait exemplaire.

62 C'est précisément l'objet de l'article de Hilde Hein dans l'ouvrage collectif sous la direction de Janet Marstine, The Routledge Companion to Museums Ethics : "Les musées, écrit-elle, ont à répondre (1) du choix de ce qu'ils montrent, ce qui inclut les moyens par lesquels ils le font; (2) de leurs non-représentations, exclusions volontaires ou non; et plus problématiquement (3) de ce qu'ils n'ont pas choisi de représenter, bien qu'ils le fassent par des moyens indirects. [...] En chaque circonstance, les musées font des choix d'ordre éthique. » (Hein, 2011, pp. 118 et 124, tr. fr. B. D.)
} 
morale, l'éthique est née de l'incertitude métaphysique et, sans postuler nécessairement le désordre universel, elle consiste à confier à l'homme le soin de définir librement ses valeurs pour ensuite mettre en œuvre les moyens de les atteindre ${ }^{63}$. Or, le musée, en tant qu'institution, réclame une éthique de tutelle, de même qu'il y a, par exemple, une éthique de l'État (la philosophie politique), une éthique de l'hôpital (la déontologie médicale), une éthique de l'école (la philosophie de l'éducation), etc. Dans chaque cas, l'éthique définit des objectifs qui orientent le fonctionnement de l'institution en posant des limites. L'institution du musée a pour effet de limiter la fonction documentaire sensible, car on ne rassemble pas et on ne montre pas n'importe quoi n'importe comment et à n'importe qui, mais on le fait toujours au nom d'un système de valeurs.

Dans un tel contexte, la nouvelle muséologie peut être comprise comme le refus de se soumettre à un ordre de valeurs imposé. Si l'on admet qu'aucun système de valeurs n'est inscrit une fois pour toutes dans la nature des choses, on peut concevoir une lutte muséale comme on a pu connaître en France une guerre scolaire (école privée/école publique), l'une et l'autre étant fondées sur un conflit entre des ordres axiologiques opposés. C'est cette guerre pour le renouvellement des valeurs muséales qui définit la nouvelle muséologie $^{64}$. La muséologie doit se défaire de toute norme morale, c'est-à-dire de toute hiérarchie des valeurs reçue de l'extérieur et vécue comme une contrainte. La nouvelle muséologie est apparue comme une protestation éthique contre un système de valeurs considéré comme étroit et usé, notamment contre une morale bourgeoise qui veut à tout prix préserver le système qui la justifie. Ces présupposés moraux, imposés de l'extérieur, privaient la muséologie de sa tâche de détermination des valeurs, car toute morale est une éthique tronquée, coupée de son pouvoir de décision, bref une idéologie qui méconnaît ses vrais fondements. Face à ce système figé, la nouvelle muséologie propose une mise en question vivante des objectifs du musée; elle est un "agent de prise de conscience » (Desvallées, 1992, p. 39). Le musée, en tant qu'institution de la fonction documentaire sensible, désormais détaché de l'obligation de sélectionner des images exemplaires ou exceptionnelles, s'ouvre à l'ensemble de la réalité naturelle et culturelle, ce qui explique l'apparition relativement récente de musées de sciences et de techniques, de musées d'anthropologie, de sites classés ou protégés, etc.

\section{Vers une muséologie contractuelle}

En tant qu'éthique, la muséologie est donc une branche de la philosophie chargée de définir des valeurs et une conduite préférables dans le cadre du rapport spécifique de l'homme avec la réalité qui constitue le champ muséal. Or c'est bien dans un esprit contractuel que le Code de déontologie de I'ICOM a été adopté en 1986, après l'Éthique des acquisitions en 1970. II met en œuvre de façon exemplaire cette mission de la muséologie ${ }^{65}$.

\footnotetext{
${ }^{63}$ La différence entre éthique et morale, qui opposa dans l'Antiquité Aristote à Platon, a été précisée et développée notamment par Gilles Deleuze dans ses travaux sur Spinoza.

${ }_{64}^{64}$ Pour l'avènement de laquelle ont lutté notamment Georges-Henri Rivière, Hugues de Varine et André Desvallées, mais aussi Marc A. Maure et bien d'autres encore.

${ }^{65}$ Le Code de déontologie de l'ICOM a été adopté à l'unanimité par la $15^{\mathrm{e}}$ Assemblée générale de l'ICOM, réunie à Buenos-Aires (Argentine) le 4 novembre 1986, modifié par la $20^{\mathrm{e}}$ Assemblée générale à Barcelone (Espagne) le 6 juillet 2001 sous le titre
} 
«La muséologie, dans ses fondements, explique François Mairesse, ne devrait pas inclure de définition a priori du mode de relation entre l'homme et la réalité, sous peine d'être définitivement figée ou de se voir dépassée. Plus rapidement qu'on ne le pense. " (Mairesse, 1999, p. 67). En effet, si aucun ordre de valeurs n'est inscrit une fois pour toutes dans la nature des choses, c'est à nous de l'inventer en fonction de nos aspirations et de nos urgences. Néanmoins, dans la mesure où il s'agit d'un rapport spécifique de l'homme avec la réalité, tous les hommes sont concernés, et il leur appartient de fonder ensemble cet ordre de valeurs, tout comme il leur appartient aussi de fonder dans le champ politique un ordre de justice sociale et de paix. Voilà qui légitime l'apparition historique d'institutions coordinatrices comme l'ICOM et plus particulièrement l'ICOFOM, qui n'ont d'autre objectif que de mettre en chantier ce cahier des charges commun. C'est pourquoi j'ai proposé l'élaboration d'une "muséologie contractuelle ». Comme on a pu parler jadis avec Rousseau de contrat social et plus récemment avec Michel Serres de contrat naturel, il est permis également de concevoir un contrat muséal, c'est-à-dire un consensus. Aussi n'avons-nous pas à nous demander ce qu'est la muséologie, car elle ne sera que ce que nous la ferons. Bref, pour reprendre les termes d'André Desvallées en 1980, "il appartient aux gens de musée de préciser s'ils souhaitent appliquer le terme de muséologie au seul langage qui leur sert à communiquer avec le public ou à l'ensemble des recherches et des réflexions qui leur permettent d'exercer pleinement leur métier, même si la plus grande partie de ces derniers se situe dans le cadre de sciences qui existeraient de toutes façons sans le musée. " (Desvallées, 1980, p. 18).

On peut comparer le système des valeurs muséales au droit naturel, qui, dans un contexte éthique, s'est révélé comme une référence conventionnelle commune à un groupe. Les jurisconsultes invoquaient jadis le droit naturel - comme un droit réputé imprescriptible présumé inscrit dans la nature de l'homme - pour justifier les dispositions adoptées par le droit positif, c'est-à-dire par l'ensemble des institutions juridiques et politiques dans un pays donné à une époque donnée. Aujourd'hui, dans une perspective éthique, on considère que le droit naturel est historique, c'est-à-dire réformable, et qu'il évolue en fonction des urgences perçues à un moment ou à un autre par la société (par exemple, la citoyenneté des femmes et le droit de tous au travail font désormais partie du droit naturel, ce qui n'était pas le cas au $\mathrm{XIX}^{\mathrm{e}}$ siècle et durant une partie du $X X^{e}$ ). II en va de même pour le musée, dont l'évolution historique s'est trouvée marquée par une série de débats et de choix successifs.

Ainsi peut-on observer des évolutions considérables dans la muséographie et dans les orientations du musée, évolutions qui témoignent indirectement des mutations opérées dans les priorités reconnues par la muséologie. Des questions concrètes, toujours chargées d'enjeux, se sont posées au cours de l'histoire du musée. On peut relever ici quelques-unes de ces acquisitions irréversibles, dont chacune correspond à la reconnaissance d'une valeur nouvelle dont le musée prend la charge. En voici quelques illustrations: l'importance de l'éclairage comme moyen de montrer tout à tous (Soufflot et l'éclairage zénithal de la grande galerie du Louvre), la

Code de déontologie de l'ICOM pour les musées et révisé par la $21^{\mathrm{e}}$ Assemblée générale à Séoul (République de Corée) le 8 octobre 2004. 
logique de l'ordre de présentation (Louis Sébastien Mercier et le Cabinet du roi, Chrétien de Mechel et la Galerie impériale et royale de Vienne), la reconstitution du contexte associée au rassemblement et à la protection des musealia, fussent-ils à l'état de fragments, (Lenoir et le Musée des monuments français), le maintien des objets sur leur site (Quatremère de Quincy et Rome la ville-musée), l'ouverture au public (Colbert, Lafont de Saint-Yenne), la propriété publique des biens culturels (Vicq d'Azyr et Poirier, la réappropriation patrimoniale), le rôle des substituts et la diffusion par l'image (Cassiano dal Pozzo, Clarac, Malraux et les diverses figures du musée imaginaire), le débat sur la restauration des objets (Violletle-Duc, Mérimée, Malraux) et, plus récemment, le toucher des expôts par le public (McLuhan et Parker et le musée non linéaire), les expositions temporaires (démocratisation de l'accès et renouvellement des expôts), l'ouverture à de nouveaux domaines, naturel, anthropologique et technique notamment, et à de nouvelles cultures (élargissement du champ des publics et des musealia), la prise en compte de la globalité des problèmes dans le temps et l'espace (Georges-Henri Rivière et l'écomusée), l'introduction de la dimension sociale et communautaire avec le rôle joué par le public (Hugues de Varine et les musées d'initiative communautaire), la prise en compte des problèmes concrets de la vie contemporaine (Jacques Hainard, Michel Colardelle, Michel Côté et les musées de société). Chacune de ces acquisitions est datée et une bonne part d'entre elles remonte seulement au $X X^{e}$ siècle, qui a découvert que le musée pouvait servir de levier social.

Dans cette histoire muséologique, qui se traduit le plus souvent sous forme d'applications muséographiques, on observe un cheminement obstiné vers l'ouverture et l'éclatement sous toutes leurs formes ${ }^{66}$. C'est ainsi que la société a valorisé successivement divers aspects, qui constituent autant d'étapes dans une histoire digne de ce nom, c'est-à-dire dans une histoire réellement novatrice.

II reste à savoir maintenant quelle figure concrète va prendre ce contrat muséal au $\mathrm{XXI}^{\mathrm{e}}$ siècle, mais il est difficile d'anticiper une série d'inventions, car une invention n'est jamais contenue dans ce qui la précède, même si elle s'y enracine nécessairement. Sans qu'il soit possible de préjuger des choix qui seront faits, deux remarques semblent s'imposer cependant : (1) d'abord rien ne nous contraint à penser qu'il s'agit d'un processus linéaire, les orientations pouvant indiquer différentes directions, des finalités multiples, parfois même contradictoires, comme cela s'est produit dans le passé notamment avec l'opposition entre Quatremère de Quincy, défenseur de la préservation in situ, et Alexandre Lenoir, l'un des artisans de la conservation in vitro. L'histoire a finalement donné raison aux deux démarches. Plus près de nous, ce fut le conflit entre la nécessaire reconnaissance des identités ethniques et l'ouverture de toutes les cultures à tous. Ces paradoxes, signes de vitalité de la muséologie, ont été relevés notamment par André Desvallées, à qui l'ICOFOM a récemment rendu hommage; (2) d'autre part, pour autant qu'elle s'éloigne des disciplines de connaissance, la muséologie ne saurait se contenter de faire des choix de valeurs dans l'abstrait sous forme

\footnotetext{
${ }^{66}$ On consultera sur ce point avec profit l'ouvrage collectif publié sous la direction de Janet Marstine, The Routledge Companion to Museum Ethics. Pour dessiner les contours de l'éthique muséale, Hilde Hein s'inspire notamment de la lutte en faveur de l'émancipation de la femme: "J'ai défendu ailleurs l'idée que la théorie féministe devrait être un guide pour re-comprendre le musée. » (p. 112). En effet, la théorie féministe, bien qu'elle ne soit pas encore adulte, a permis de prendre conscience de certaines inerties de notre civilisation occidentale et de lutter contre elles.
} 
théorique, mais elle ne peut que conduire à l'action. Certes il n'est pas possible d'agir de façon efficace et réfléchie sans avoir fait des choix préalables, mais définir des valeurs sans qu'elles débouchent sur une action concrète est totalement vain. La muséologie contractuelle est donc indissociable d'une transformation effective de l'institution et réclame de toute urgence une mise en chantier. Si quelqu'un d'un peu plus jeune que moi voulait en prendre la charge, en créant un laboratoire expérimental, une sorte d'OuMuPo, c'est-àdire un " ouvroir de muséologie potentielle " - si l'on permet cette allusion au groupe fondé en 1960 par Raymond Queneau et François Le Lionnais -, afin de reconsidérer l'entreprise dans sa totalité sur la base d'une réelle concertation, il rendrait probablement le plus grand service que puisse attendre le monde des musées. Mais je sais qu'il est infiniment peu probable que je sois suivi dans cette démarche assurément dérangeante.

\section{Références}

Deloche, B. (1999). "Muséologie et philosophie, Provocative Paper ", Icofom Study Series (Muséologie et philosophie), 31, 8-17.

Deloche, B. (2001). Le Musée virtuel. Vers une éthique des nouvelles images, Paris : PUF.

Desvallées, A. (1980). «La muséologie - science ou seulement travail pratique du musée ", DoTraM-MuWoP, $\mathrm{n}^{\circ} 1,17-18$.

Desvallées, A. (1987). « Un tournant de la muséologie », Brises, 10, 5-12.

Desvallées, A. (1994). "Les musées d'ethnographie ont-ils encore un sens ? " Anales del museo nacional de antropologia, $\mathrm{n}^{\circ} 1$, Madrid.

Desvallées, A. (Ed.). (1992). Vagues 1. Une anthologie de la nouvelle muséologie, Mâcon, W/MNES, 1992.

Ecole du Louvre (1983). Quels musées, pour quelles fins, aujourd'hui ? séminaire de l'École du Louvre, mars 1983, Paris: La Documentation française.

Gregorova, A. (1980). La muséologie - science ou seulement travail pratique du musée, DoTraM-MuWoP, $\mathrm{n}^{\circ} 1,19-21$.

Hein, H. (2011). "The responsibility of Representation. A feminist perspective. " Dans J. Marstine. The Routledge Companion Museum Ethics, Oxon, Routledge.

Hudson, K. (1997). "Who are the 'museologists' and for whose benefit do they exist? " Dans Z.Z. Stransky (Ed.), Museology for Tomorrow's World (pp. 102-110). Munich, Germany: Verlag Dr. C. MüllerStraten (tr. fr. F. Mairesse et A. Desvallées).

Le Code de déontologie de I'ICOM pour les musées (1986-2004). Paris: ICOM (différentes versions successives de 1986 à 2004).

Mairesse, F. (1999) "La relation spécifique. " ICOFOM Study Series: Muséologie et philosophie, 31, 60-67.

Rivière, G.H. et al. (1989). La Muséologie selon Georges-Henri Rivière, Paris : Dunod.

Spielbauer, J. (1987). "Museums and Museology: a Means to Active Integrative Preservation », Icofom Study Series, 12, 271-277.

Stránský, Z. Z. (1980). « La muséologie - science ou seulement travail pratique du musée ", DoTraM-MuWoP, $\mathrm{n}^{\circ} 1,42-44$.

Stránský, Z. Z. (1986). «Avant-propos méthodologique », Workshop ICOFOM, Berlin, 16-22 mai (non publié). 


\section{Résumé}

La muséologie manque de définition précise sur son champ de savoir. Divers propositions ont été faites ; l'auteur examine d'abord le champ muséal du point de vue spécifique sur la réalité, mais ce champ muséal n'est pas de l'ordre de la connaissance. Moins bien cerné que celui du musée lui-même, le champ de la muséologie est d'ordre éthique. II propose finalement de suivre la voie d'une muséologie contractuelle dont il donne les paramètres.

Mots clé : muséologie, définition de la muséologie

\section{Abstract}

\section{In favour of a contractual museology}

Museology lacks a precise definition of its field of knowledge. Several proposals have been made; the author first examines the concept of the museum field from the approach of a specific view of reality, but this museum field is not within the realm of knowledge. Less well defined than the museum itself, the museological field is in the realm of ethics. The author finally suggests following the path of contractual museology and defines the sphere.

Key words: museology, definition of museology 